\title{
Lack of HLA predominance and HLA shared epitopes in biliary Atresia
}

\author{
Cara L Mack ${ }^{1,3^{*}}$, Kirsten M Anderson ${ }^{1}$, Michael T Aubrey ${ }^{2}$, Philip Rosenthal ${ }^{4}$, Ronald J Sokol ${ }^{3}$ and Brian M Freed ${ }^{1,2}$
}

\begin{abstract}
Biliary atresia (BA) is characterized by progressive inflammation and fibrosis of bile ducts. A theory of pathogenesis entails autoimmune-mediated injury targeting bile duct epithelia. One of the strongest genetic associations with autoimmunity is with HLA genes. In addition, apparently dissimilar HLA alleles may have similar antigen-binding sites, called shared epitopes, that overlap in their capacity to present antigens. In autoimmune disease, the incidence of the disease may be related to the presence of shared epitopes, not simply the HLA allelic association. Aim: To determine HLA allele frequency (high-resolution genotyping) and shared epitope associations in BA. Results: Analysis of every allele for HLA-A, -B, -C, -DRB1, -DPB1 and -DQB1 in 180 BA and 360 racially-matched controls did not identify any significant HLA association with BA. Furthermore, shared epitope analysis of greater than 10 million possible combinations of peptide sequences was not different between BA and controls.

Conclusions: This study encompasses the largest HLA allele frequency analysis for BA in the United States and is the first study to perform shared epitope analysis. When controlling for multiple comparisons, no HLA allele or shared epitope association was identified in BA. Future studies of genetic links to BA that involve alterations of the immune response should include investigations into defects in regulatory T cells and non-HLA linked autoinflammatory diseases.
\end{abstract}

Biliary atresia is the most common neonatal cholestatic disorder, occurring in approximately 1 in 10,000-15,000 live births in the United States. The disease is characterized by complete fibrotic obliteration of the lumen of all or part of the extrahepatic biliary tree within three months of life and progressive inflammation and fibrosis of intrahepatic bile ducts (Sokol et al. 2003). There are two proposed types of $\mathrm{BA}$; the embryonic form and the perinatal form. The embryonic form ( $15 \%$ of cases) may be due to defective development of the extrahepatic biliary tract and is associated with other congenital anomalies. The perinatal or acquired form occurs in $\sim 85 \%$ of cases and the bile duct damage has been theorized to be due to an initial virus infection of the biliary tree that triggers a secondary autoimmunemediated injury targeting bile duct epithelia. The continued autoimmune response would lead to progressive

\footnotetext{
*Correspondence: Cara.Mack@ucdenver.edu

1 Departments of Medicine and Immunology, Division of Allergy and Clinical Immunology, University of Colorado School of Medicine, Aurora, CO 80045, USA

${ }^{3}$ Department of Pediatrics, Division of Pediatric Gastroenterology, Digestive Health Institute, Children's Hospital Colorado, Hepatology and Nutrition, 13123 East 16th Ave. B290, Aurora, CO 80045, USA

Full list of author information is available at the end of the article
}

intrahepatic bile duct injury and sclerosis, resulting in cirrhosis (Mack et al. 2007). Despite clinical improvement following a portoenterostomy procedure at the time of diagnosis, up to $80 \%$ of children with BA will eventually require liver transplantation (Sokol et al. 2003).

Compelling evidence for autoimmunity has been gained from mouse studies, where autoreactive $\mathrm{T}$ cells targeting bile duct epithelia have been identified. Two groups have demonstrated that autoreactive $\mathrm{T}$ cells specific to bile duct epithelia are present in the rotavirus-induced mouse model of BA and are associated with bile duct inflammation and injury (Mack et al. 2006; Shivakumar et al. 2007). In vitro analyses demonstrated significant increases in liver $\mathrm{T}$ cells from BA mice that generated IFN- $\gamma$ in response to either virus or self-bile duct epithelial antigens (Mack et al. 2006). In addition, adoptive transfer of liver T cells from BA mice into immunodeficient recipients led to bile duct-specific inflammation and injury (Mack et al. 2006; Shivakumar et al. 2007). This induction of bile duct pathology occurred in the absence of detectable transferred virus, suggesting that bile duct antigens were the target of the $\mathrm{T}$ cells. Human studies pertaining to autoimmunity in BA are limited. Identification of oligoclonal populations,

\section{实}

(c) 2013 Mack et al.; licensee Springer. This is an Open Access article distributed under the terms of the Creative Commons Attribution License (http://creativecommons.org/licenses/by/2.0), which permits unrestricted use, distribution, and reproduction in any medium, provided the original work is properly cited. 
defined as $\mathrm{T}$ cells expressing similar $\mathrm{T}$ cell receptor variable regions of the $\beta$-chain, from BA livers at the time of diagnosis suggests specific antigen-driven inflammation (Mack et al. 2007). It remains unknown what the potential antigens are (e.g. viral proteins, bile duct epithelial proteins) that are responsible for $\mathrm{T}$ cell activation and bile duct injury.

One of the strongest genetic associations with autoimmunity is with the human leukocyte antigen (HLA) genes. HLA is a region on chromosome 6 that contains more than 50 genes known to be involved in the immune response. HLA class I (HLA-A,-B and -C) are single polypeptide chains that present endogenous peptides to $\mathrm{CD}^{+}$ $\mathrm{T}$ cells and HLA class II molecules (HLA-DR, -DP and -DQ) are heterodimers expressed predominantly on hematopoietic cells that present exogenous peptides to $\mathrm{CD}^{+}$helper $\mathrm{T}$ cells (Abbas \& Lichtman 2000). HLA associations with BA have been reported with conflicting results (Silveira et al. 1993; A-Kader et al. 2002; Donaldson et al. 2002; Yuasa et al. 2005), mainly due to the sample size analyzed and the level of resolution used to identify the HLA alleles.

The vast majority of HLA polymorphisms are located within the antigen-binding cleft of the HLA molecule that comes in contact with the peptide or T cell receptor. Peptide binding to HLA-DRB1 molecules, for example, is controlled by 6 pockets within the cleft, each pocket with multiple polymorphic amino acids that create millions of potential peptide binding epitopes (Stern et al. 1994). The peptide fits into a binding groove in the HLA molecule; the residues that are available to interact with peptides in the HLA are located in this groove, and are found to be highly polymorphic among the population, with different residues corresponding to different HLA alleles. Apparently dissimilar HLA alleles may have similar antigenbinding sites, called shared epitopes, and thereby overlap in their capacity to present antigens (Gregersen et al. 1987). Thus in autoimmune and immune-mediated diseases, the incidence and severity of the disease may be related to the presence of shared epitopes, not simply the HLA allelic association (Klareskog et al. 2004).

In this study we sought to determine HLA allele frequency and shared epitope associations in BA patients in the United States, through high-resolution HLA genotyping of all class I and II alleles as well as shared epitope analysis. Identification of potential HLA associations with BA would provide clues to the immune-mediated pathogenesis of this disease.

\section{Methods}

Study subjects

Biliary atresia

Peripheral blood samples were obtained from participants already enrolled in two ongoing NIDDK-funded clinical studies that are being conducted through the Childhood Liver Disease Research and Education Network (ChiLDREN). The NIDDK-funded repository at Rutger's University processed the blood samples and either stored the DNA directly or developed EBV transformed cell lines, followed by DNA extraction. Our study utilized DNA samples from patients with the perinatal/acquired form of $\mathrm{BA}$, excluding those BA subjects with biliary atresia splenic malformation syndrome and those with other major congenital malformations. Information obtained at the time of sample collection or during the course of the research studies included: sex, race, ethnicity, age at sample collection, age at liver transplant, and outcome (alive with native liver, death or transplant). A total of $178 \mathrm{BA}$ patient DNA samples were available for analysis (Table 1): 76 patients $(42.7 \%)$ had undergone liver transplant or died within the first two years of life (severe course); 71 patients (39.9\%) were 5 years of age or older and had not yet received a liver transplant (mild course); and 31 (17.4\%)

Table 1 Racial distribution of subjects

\begin{tabular}{|c|c|c|}
\hline Race & BA $n(\%)$ & Controls $n(\%)$ \\
\hline \multicolumn{3}{|l|}{ HLA-A,B } \\
\hline Black & $25(14.2)$ & $47(14.3)$ \\
\hline Caucasian & $114(64.8)$ & $213(64.7)$ \\
\hline Hispanic & $23(13.1)$ & $43(13.1)$ \\
\hline Asian & $14(8.0)$ & $26(7.9)$ \\
\hline Total & 176 & 329 \\
\hline \multicolumn{3}{|l|}{ HLA-C } \\
\hline Black & $25(14.1)$ & $50(14.3)$ \\
\hline Caucasian & $115(65.0)$ & $226(64.6)$ \\
\hline Hispanic & $23(13.0)$ & $46(13.1)$ \\
\hline Asian & $14(7.9)$ & $28(8.0)$ \\
\hline Total & 177 & 350 \\
\hline \multicolumn{3}{|l|}{ HLA-DP } \\
\hline Caucasian & $111(100)$ & $91(100)$ \\
\hline \multicolumn{3}{|l|}{ HLA-DQ } \\
\hline Black & $25(14.0)$ & $48(14.0)$ \\
\hline Caucasian & $116(65.2)$ & $223(65.2)$ \\
\hline Hispanic & $23(12.9)$ & 44 (12.9) \\
\hline Asian & $14(7.9)$ & $27(7.9)$ \\
\hline Total & 178 & 342 \\
\hline \multicolumn{3}{|l|}{ HLA-DR } \\
\hline Black & $25(14.0)$ & $96(14.0)$ \\
\hline Caucasian & $116(65.2)$ & $446(65.2)$ \\
\hline Hispanic & $23(12.9)$ & $88(12.9)$ \\
\hline Asian & $14(7.9)$ & $54(7.9)$ \\
\hline Total & 178 & 684 \\
\hline
\end{tabular}


Table 2 HLA-A single amino acid polymorphisms**

\begin{tabular}{|c|c|c|c|c|c|c|}
\hline Positions $^{\dagger}$ & Amino acid & Patients (176) n (\%) & Controls (329) n (\%) & Odds Ratio & $P u$ value* & $P c$ value* \\
\hline 9 & $\mathrm{~F}$ & 134(76.14) & $251(76.29)$ & 1.0 & 0.97 & NS \\
\hline 9 & $S$ & $47(26.70)$ & 108(32.83) & 0.8 & 0.16 & NS \\
\hline 9 & $\mathrm{~T}$ & $31(17.61)$ & $55(16.72)$ & 0.9 & 0.80 & NS \\
\hline 9 & Y & 70(39.77) & 123(37.39) & 0.9 & 0.60 & NS \\
\hline 44 & K & $44(25.00)$ & $84(25.53)$ & 1.0 & 0.90 & NS \\
\hline 56 & $\mathrm{R}$ & $21(11.93)$ & $39(11.85)$ & 1.0 & 0.98 & NS \\
\hline 62 & $E$ & $33(18.75)$ & $89(27.05)$ & 0.7 & 0.04 & NS \\
\hline 62 & G & $82(46.59)$ & $160(48.63)$ & 1.0 & 0.66 & NS \\
\hline 62 & Q & $113(64.20)$ & 197(59.88) & 0.9 & 0.34 & NS \\
\hline 62 & $\mathrm{R}$ & $57(32.39)$ & $94(28.57)$ & 0.9 & 0.37 & NS \\
\hline 63 & $\mathrm{~N}$ & $57(32.39)$ & $94(28.57)$ & 0.9 & 0.37 & NS \\
\hline 65 & G & $32(18.18)$ & $89(27.05)$ & 0.7 & 0.03 & NS \\
\hline 66 & K & 104(59.09) & $223(67.78)$ & 0.9 & 0.05 & NS \\
\hline 66 & $\mathrm{~N}$ & 152(86.36) & $257(78.12)$ & 0.9 & 0.02 & NS \\
\hline 67 & M & $44(25.00)$ & $84(25.53)$ & 1.0 & 0.90 & NS \\
\hline 70 & $\mathrm{H}$ & 157(89.20) & $310(94.22)$ & 0.9 & 0.04 & NS \\
\hline 70 & Q & $92(52.27)$ & 164(49.85) & 1.0 & 0.60 & NS \\
\hline 73 & 1 & $20(11.36)$ & $37(11.25)$ & 1.0 & 0.97 & NS \\
\hline 74 & D & $163(92.61)$ & 296(89.97) & 1.0 & 0.33 & NS \\
\hline 74 & $\mathrm{H}$ & $82(46.59)$ & $160(48.63)$ & 1.0 & 0.66 & NS \\
\hline 76 & A & $66(37.50)$ & $112(34.04)$ & 0.9 & 0.44 & NS \\
\hline 76 & $E$ & $55(31.25)$ & 119(36.17) & 0.9 & 0.27 & NS \\
\hline 76 & V & $151(85.80)$ & $270(82.07)$ & 1.0 & 0.28 & NS \\
\hline 77 & $D$ & $151(85.80)$ & $270(82.07)$ & 1.0 & 0.28 & NS \\
\hline 77 & $\mathrm{~N}$ & 102(57.95) & 187(56.84) & 1.0 & 0.81 & NS \\
\hline 79 & $\mathrm{R}$ & $46(26.14)$ & $111(33.74)$ & 0.8 & 0.08 & NS \\
\hline 80 & 1 & $46(26.14)$ & $111(33.74)$ & 0.8 & 0.08 & NS \\
\hline 81 & A & $46(26.14)$ & $111(33.74)$ & 0.8 & 0.08 & NS \\
\hline 82 & $\mathrm{~L}$ & $46(26.14)$ & $111(33.74)$ & 0.8 & 0.08 & NS \\
\hline 83 & $\mathrm{R}$ & $46(26.14)$ & $111(33.74)$ & 0.8 & 0.08 & NS \\
\hline 90 & D & $85(48.30)$ & $150(45.59)$ & 0.9 & 0.56 & NS \\
\hline 95 & 1 & 152(86.36) & 259(78.72) & 0.9 & 0.04 & NS \\
\hline 95 & $L$ & $37(21.02)$ & 104(31.61) & 0.7 & 0.01 & NS \\
\hline 95 & V & $76(43.18)$ & $145(44.07)$ & 1.0 & 0.85 & NS \\
\hline 97 & 1 & 109(61.93) & 184(55.93) & 0.9 & 0.19 & NS \\
\hline 97 & M & $74(42.05)$ & 175(53.19) & 0.8 & 0.02 & NS \\
\hline 97 & $\mathrm{R}$ & $99(56.25)$ & 198(60.18) & 0.9 & 0.39 & NS \\
\hline 99 & $\mathrm{~F}$ & $32(18.18)$ & $89(27.05)$ & 0.7 & 0.03 & NS \\
\hline 105 & $P$ & $92(52.27)$ & $167(50.76)$ & 1.0 & 0.75 & NS \\
\hline 105 & S & 158(89.77) & 297(90.27) & 1.0 & 0.86 & NS \\
\hline 107 & G & 164(93.18) & 296(89.97) & 1.0 & 0.23 & NS \\
\hline 107 & W & $82(46.59)$ & $161(48.94)$ & 1.0 & 0.62 & NS \\
\hline 114 & $\mathrm{H}$ & 108(61.36) & $232(70.52)$ & 0.9 & 0.04 & NS \\
\hline 114 & Q & $48(27.27)$ & $85(25.84)$ & 0.9 & 0.73 & NS \\
\hline
\end{tabular}


Table 2 HLA-A single amino acid polymorphisms** (Continued)

\begin{tabular}{|c|c|c|c|c|c|c|}
\hline 114 & $\mathrm{R}$ & 109(61.93) & 193(58.66) & 0.9 & 0.48 & NS \\
\hline 116 & D & $142(80.68)$ & $241(73.25)$ & 0.9 & 0.06 & NS \\
\hline 116 & Y & 108(61.36) & $232(70.52)$ & 0.9 & 0.04 & NS \\
\hline 127 & K & $115(65.34)$ & 250(75.99) & 0.9 & 0.01 & NS \\
\hline 127 & N & $143(81.25)$ & $243(73.86)$ & 0.9 & 0.06 & NS \\
\hline 142 & I & 157(89.20) & 284(86.32) & 1.0 & 0.35 & NS \\
\hline 142 & $\mathrm{~T}$ & $94(53.41)$ & $191(58.05)$ & 0.9 & 0.32 & NS \\
\hline 144 & Q & $77(43.75)$ & 138(41.95) & 1.0 & 0.70 & NS \\
\hline 145 & $\mathrm{H}$ & $94(53.41)$ & $191(58.05)$ & 0.9 & 0.32 & NS \\
\hline 145 & $\mathrm{R}$ & 157(89.20) & $284(86.32)$ & 1.0 & 0.35 & NS \\
\hline 149 & $\mathrm{~T}$ & 28(15.91) & $41(12.46)$ & 0.8 & 0.28 & NS \\
\hline 150 & V & $44(25.00)$ & $84(25.53)$ & 1.0 & 0.90 & NS \\
\hline 151 & R & $59(33.52)$ & $112(34.04)$ & 1.0 & 0.91 & NS \\
\hline 152 & A & $57(32.39)$ & 119(36.17) & 0.9 & 0.40 & NS \\
\hline 152 & $E$ & $65(36.93)$ & $96(29.18)$ & 0.8 & 0.07 & NS \\
\hline 152 & v & $140(79.55)$ & $291(88.45)$ & 0.9 & 0.01 & NS \\
\hline 156 & L & $143(81.25)$ & $257(78.12)$ & 1.0 & 0.41 & NS \\
\hline 156 & Q & $40(22.73)$ & $101(30.70)$ & 0.7 & 0.06 & NS \\
\hline 156 & R & $44(25.00)$ & $84(25.53)$ & 1.0 & 0.90 & NS \\
\hline 156 & W & $49(27.84)$ & $93(28.27)$ & 1.0 & 0.92 & NS \\
\hline 158 & V & $44(25.00)$ & $84(25.53)$ & 1.0 & 0.90 & NS \\
\hline 161 & D & $44(25.00)$ & $56(17.02)$ & 0.7 & 0.03 & NS \\
\hline 163 & $\mathrm{R}$ & 79(44.89) & $144(43.77)$ & 1.0 & 0.81 & NS \\
\hline 166 & D & 73(41.48) & 155(47.11) & 0.9 & 0.23 & NS \\
\hline 166 & $E$ & 163(92.61) & 295(89.67) & 1.0 & 0.28 & NS \\
\hline 167 & G & $73(41.48)$ & $155(47.11)$ & 0.9 & 0.23 & NS \\
\hline 167 & W & $163(92.61)$ & 295(89.67) & 1.0 & 0.28 & NS \\
\hline
\end{tabular}

*Pu, uncorrected $P$ values; $P c$, corrected $P$ values.

† Polymorphic amino acid positions $3,9,12,14,17,19,31,35,43,44,56,62,63,65,66,67,70,73,74,76,77,79,80,81,82,83,90,95,97,99,102,105,107,109$, 114, 116, 127, 142, 144, 149, 150, 151, 152, 156, 158, 161, 163, 166, 167, 171.

** Not displaying amino acid residues that were present in $90 \%$ or greater in both patient and control groups, or were present in $10 \%$ or fewer in both patient and control groups.

were between the ages of 2-5 years and were with or without liver transplant (moderate course).

\section{Controls}

Access to complete HLA genotyping on over 6,600 cord blood samples from the state of Colorado was available through ClinImmune Labs. Two control samples were analyzed for every one BA sample $(n=329-350)$ with the exceptions of HLA-DP $(\mathrm{n}=91)$ and HLA-DR $(\mathrm{n}=684)$. There were slight differences in the availability of various racial and ethnic groups in the control samples, therefore, racially and ethnically-balanced groups for each of the various HLA molecules were created from sequentially-selected controls subjects (Table 1).

\section{High resolution HLA genotyping}

High resolution allele typing was performed for HLA-A, HLA-B, HLA-C, HLA-DRB1, HLA-DQB1, and HLADPB1. Automated capillary electrophoresis sequencing (sequenced-based typing, SBT) was performed on all samples with fluorescent dye technologies (ABI Big Dye, version 1.1). A generic PCR amplification precedes sequencing in both directions using intronic class I primers. HLA-A,B,C sequencing covered exons 2, 3 and 4 . Class II SBT for HLA-DRB1, DQB1, and DPB1 covered exon 2. Sequences were interpreted with computer-assisted Assign Software (version 3.5) against current IMGT sequencing libraries (January 2008 to April 2010). The raw sequence electropherograms were archived for future retrieval. Every homozygous sequencing result was confirmed with 
Table 3 HLA-B single amino acid polymorphisms**

\begin{tabular}{|c|c|c|c|c|c|c|}
\hline Positions $^{\dagger}$ & Amino acid & Patients (176) n (\%) & Controls (329) n (\%) & Odds Ratio & $P u$ value* & $P c$ value ${ }^{*}$ \\
\hline 9 & $\mathrm{D}$ & $29(16.48)$ & $52(15.81)$ & 1.0 & 0.84 & NS \\
\hline 9 & $\mathrm{H}$ & $57(32.39)$ & 118(35.87) & 0.9 & 0.43 & NS \\
\hline 11 & A & $154(87.50)$ & 303(92.10) & 1.0 & 0.09 & NS \\
\hline 11 & $S$ & $85(48.30)$ & 174(52.89) & 0.9 & 0.33 & NS \\
\hline 12 & M & 149(84.66) & 298(90.58) & 0.9 & 0.05 & NS \\
\hline 12 & V & 95(53.98) & 187(56.84) & 0.9 & 0.54 & NS \\
\hline 24 & A & $92(52.27)$ & $172(52.28)$ & 1.0 & 1.00 & NS \\
\hline 24 & S & $110(62.50)$ & $205(62.31)$ & 1.0 & 0.97 & NS \\
\hline 24 & $\mathrm{~T}$ & $88(50.00)$ & $173(52.58)$ & 1.0 & 0.58 & NS \\
\hline 32 & $\mathrm{~L}$ & 79(44.89) & $162(49.24)$ & 0.9 & 0.35 & NS \\
\hline 41 & T & $80(45.45)$ & $157(47.72)$ & 1.0 & 0.63 & NS \\
\hline 45 & E & $115(65.34)$ & $210(63.83)$ & 1.0 & 0.74 & NS \\
\hline 45 & K & $72(40.91)$ & $147(44.68)$ & 0.9 & 0.42 & NS \\
\hline 45 & M & $43(24.43)$ & $83(25.23)$ & 1.0 & 0.84 & NS \\
\hline 45 & $\mathrm{~T}$ & $71(40.34)$ & $137(41.64)$ & 1.0 & 0.78 & NS \\
\hline 46 & A & $43(24.43)$ & $83(25.23)$ & 1.0 & 0.84 & NS \\
\hline 62 & G & 19(10.80) & $37(11.25)$ & 1.0 & 0.88 & NS \\
\hline 63 & $E$ & $122(69.32)$ & $245(74.47)$ & 0.9 & 0.22 & NS \\
\hline 63 & $\mathrm{~N}$ & 136(77.27) & 269(81.76) & 0.9 & 0.23 & NS \\
\hline 65 & R & $22(12.50)$ & $40(12.16)$ & 1.0 & 0.91 & NS \\
\hline 66 & $\mathrm{~N}$ & $22(12.50)$ & $40(12.16)$ & 1.0 & 0.91 & NS \\
\hline 67 & C & $49(27.84)$ & $78(23.71)$ & 1.2 & 0.31 & NS \\
\hline 67 & F & 68(38.64) & $139(42.25)$ & 0.9 & 0.43 & NS \\
\hline 67 & M & $22(12.50)$ & $40(12.16)$ & 1.0 & 0.91 & NS \\
\hline 67 & S & $110(62.50)$ & $215(65.35)$ & 1.0 & 0.52 & NS \\
\hline 67 & Y & $56(31.82)$ & $97(29.48)$ & 1.1 & 0.59 & NS \\
\hline 69 & A & $75(42.61)$ & $144(43.77)$ & 1.0 & 0.80 & NS \\
\hline 70 & Q & $55(31.25)$ & $96(29.18)$ & 1.1 & 0.63 & NS \\
\hline 70 & $S$ & $22(12.50)$ & $40(12.16)$ & 1.0 & 0.91 & NS \\
\hline 71 & A & $78(44.32)$ & $146(44.38)$ & 1.0 & 0.99 & NS \\
\hline 74 & $D$ & $107(60.80)$ & 193(58.66) & 1.0 & 0.64 & NS \\
\hline 74 & Y & $151(85.80)$ & $291(88.45)$ & 1.0 & 0.39 & NS \\
\hline 77 & $\mathrm{~N}$ & $94(53.41)$ & 183(55.62) & 1.0 & 0.63 & NS \\
\hline 77 & S & 155(88.07) & 276(83.89) & 1.0 & 0.21 & NS \\
\hline 80 & 1 & $50(28.41)$ & $106(32.22)$ & 0.9 & 0.38 & NS \\
\hline 80 & $\mathrm{~N}$ & $154(87.50)$ & 277(84.19) & 1.0 & 0.32 & NS \\
\hline 80 & $\mathrm{~T}$ & $66(37.50)$ & $118(35.87)$ & 1.0 & 0.72 & NS \\
\hline 81 & A & $94(53.41)$ & 183(55.62) & 1.0 & 0.63 & NS \\
\hline 81 & $\mathrm{~L}$ & 158(89.77) & 290(88.15) & 1.0 & 0.58 & NS \\
\hline 82 & $L$ & 104(59.09) & 197(59.88) & 1.0 & 0.86 & NS \\
\hline 82 & $\mathrm{R}$ & $154(87.50)$ & 277(84.19) & 1.0 & 0.32 & NS \\
\hline 83 & G & $154(87.50)$ & 277(84.19) & 1.0 & 0.32 & NS \\
\hline 83 & R & 104(59.09) & 197(59.88) & 1.0 & 0.86 & NS \\
\hline 94 & 1 & $85(48.30)$ & $163(49.54)$ & 1.0 & 0.79 & NS \\
\hline
\end{tabular}


Table 3 HLA-B single amino acid polymorphisms** (Continued)

\begin{tabular}{|c|c|c|c|c|c|c|}
\hline 95 & 1 & $91(51.70)$ & $166(50.46)$ & 1.0 & 0.79 & NS \\
\hline 95 & $\mathrm{~L}$ & $143(81.25)$ & $256(77.81)$ & 1.0 & 0.37 & NS \\
\hline 95 & W & 49(27.84) & 106(32.22) & 0.9 & 0.31 & NS \\
\hline 97 & R & 139(78.98) & $245(74.47)$ & 1.1 & 0.26 & NS \\
\hline 97 & $S$ & $80(45.45)$ & $145(44.07)$ & 1.0 & 0.77 & NS \\
\hline 97 & $\mathrm{~T}$ & $35(19.89)$ & $83(25.23)$ & 0.8 & 0.18 & NS \\
\hline 97 & W & 24(13.64) & 23(6.99) & 2.0 & 0.01 & NS \\
\hline 103 & $\mathrm{~L}$ & 70(39.77) & 103(31.31) & 1.3 & 0.06 & NS \\
\hline 113 & Y & $85(48.30)$ & $147(44.68)$ & 1.1 & 0.44 & NS \\
\hline 114 & D & 133(75.57) & $241(73.25)$ & 1.0 & 0.57 & NS \\
\hline 114 & N & $122(69.32)$ & $227(69.00)$ & 1.0 & 0.94 & NS \\
\hline 116 & D & $52(29.55)$ & 103(31.31) & 0.9 & 0.68 & NS \\
\hline 116 & $F$ & $47(26.70)$ & $67(20.36)$ & 1.3 & 0.10 & NS \\
\hline 116 & $\mathrm{~L}$ & 28(15.91) & $48(14.59)$ & 1.1 & 0.69 & NS \\
\hline 116 & $S$ & $72(40.91)$ & $135(41.03)$ & 1.0 & 0.98 & NS \\
\hline 116 & Y & 108(61.36) & $221(67.17)$ & 0.9 & 0.19 & NS \\
\hline 131 & $\mathrm{R}$ & $89(50.57)$ & $173(52.58)$ & 1.0 & 0.67 & NS \\
\hline 131 & S & 158(89.77) & 299(90.88) & 1.0 & 0.69 & NS \\
\hline 143 & $S$ & 14(7.95) & $37(11.25)$ & 0.7 & 0.24 & NS \\
\hline 147 & L & 14(7.95) & $37(11.25)$ & 0.7 & 0.24 & NS \\
\hline 152 & $E$ & 108(61.36) & 188(57.14) & 1.1 & 0.36 & NS \\
\hline 152 & V & 155(88.07) & $281(85.41)$ & 1.0 & 0.41 & NS \\
\hline 156 & D & $63(35.80)$ & 111(33.74) & 1.1 & 0.64 & NS \\
\hline 156 & $\mathrm{~L}$ & 148(84.09) & 275(83.59) & 1.0 & 0.88 & NS \\
\hline 156 & R & $40(22.73)$ & $80(24.32)$ & 0.9 & 0.69 & NS \\
\hline 156 & W & $24(13.64)$ & $37(11.25)$ & 1.2 & 0.43 & NS \\
\hline 158 & $\mathrm{~T}$ & 17(9.66) & $33(10.03)$ & 1.0 & 0.89 & NS \\
\hline 163 & $E$ & $71(40.34)$ & 149(45.29) & 0.9 & 0.29 & NS \\
\hline 163 & L & 123(69.89) & $248(75.38)$ & 0.9 & 0.18 & NS \\
\hline 163 & $\mathrm{~T}$ & $87(49.43)$ & $148(44.98)$ & 1.1 & 0.34 & NS \\
\hline 167 & $S$ & $46(26.14)$ & $87(26.44)$ & 1.0 & 0.94 & NS \\
\hline 171 & $\mathrm{H}$ & $46(26.14)$ & $93(28.27)$ & 0.9 & 0.61 & NS \\
\hline 177 & D & $81(46.02)$ & $162(49.24)$ & 0.9 & 0.49 & NS \\
\hline 178 & K & $50(28.41)$ & 109(33.13) & 0.9 & 0.28 & NS \\
\hline 180 & $E$ & $81(46.02)$ & $162(49.24)$ & 0.9 & 0.49 & NS \\
\hline
\end{tabular}

${ }^{*} P u$, uncorrected $P$ values; $P c$, corrected $P$ values.

† Polymorphic amino acid positions $3,9,12,14,17,19,31,35,43,44,56,62,63,65,66,67,70,73,74,76,77,79,80,81,82,83,90,95,97,99,102,105,107,109$, $114,116,127,142,144,149,150,151,152,156,158,161,163,166,167,171$.

** Not displaying amino acid residues that were present in $90 \%$ or greater in both patient and control groups, or were present in $10 \%$ or fewer in both patient and control groups.

a secondary technology, such as Luminex SSO (One Lambda) or PCR SSP (Life Technologies).

\section{Sample size calculation for HLA genotyping}

Power calculations, based on Chi square test with continuity correction, without correction for multiple comparisons, was used to determine the sample size required to find a statistically significant difference between a control group frequency of an HLA allele and the BA frequency with a two-sided test using power of 0.8 and a significance level of 0.05 . In order to detect a $10 \%$ increased frequency of a given HLA allele in BA versus control (i.e. $35 \%$ frequency in BA versus $25 \%$ in controls), a minimum of 157 patient samples was required. 
Table 4 HLA-C single amino acid polymorphisms

\begin{tabular}{|c|c|c|c|c|c|c|}
\hline Position** & Amino acid & $\mathrm{BA}(177) n(\%)$ & Controls (350) $n(\%)$ & Odds Ratio & $P u$ value ${ }^{\#}$ & $P c$ value \\
\hline 9 & $S$ & $43(24.3)$ & 109(31.1) & 0.78 & 0.12 & NS \\
\hline 9 & D & 106(59.9) & $190(54.3)$ & 1.10 & 0.17 & NS \\
\hline 9 & Y & $125(70.6)$ & $236(67.4)$ & 1.05 & 0.35 & NS \\
\hline 11 & $S$ & $56(31.6)$ & $132(37.7)$ & 0.84 & 0.20 & NS \\
\hline 14 & W & $39(22.0)$ & $98(28.0)$ & 0.79 & 0.16 & NS \\
\hline 21 & $\mathrm{H}$ & $61(34.5)$ & $117(33.4)$ & 1.03 & 0.74 & NS \\
\hline 24 & $S$ & $116(65.5)$ & 208(59.4) & 1.10 & 0.13 & NS \\
\hline 24 & A & $149(84.2)$ & $290(82.9)$ & 1.02 & 0.50 & NS \\
\hline 35 & Q & $49(27.7)$ & $92(26.3)$ & 1.05 & 0.68 & NS \\
\hline 49 & $E$ & $39(22.0)$ & $98(28.0)$ & 0.79 & 0.16 & NS \\
\hline 66 & $\mathrm{~N}$ & $48(27.1)$ & $96(27.4)$ & 0.99 & 1.00 & NS \\
\hline 73 & $\mathrm{~T}$ & $113(63.8)$ & $230(65.7)$ & 0.97 & 0.80 & NS \\
\hline 73 & A & $136(76.8)$ & $277(79.1)$ & 0.97 & 0.71 & NS \\
\hline 77 & $\mathrm{~N}$ & $106(59.9)$ & $227(64.9)$ & 0.92 & 0.34 & NS \\
\hline 77 & $S$ & 153(86.4) & 294(84.0) & 1.03 & 0.30 & NS \\
\hline 80 & K & 106(59.9) & $227(64.9)$ & 0.92 & 0.34 & NS \\
\hline 80 & $N$ & 153(86.4) & $294(84.0)$ & 1.03 & 0.30 & NS \\
\hline 90 & D & $125(70.6)$ & $255(72.9)$ & 0.97 & 0.73 & NS \\
\hline 90 & A & 129(72.9) & $257(73.4)$ & 0.99 & 0.94 & NS \\
\hline 91 & $\mathrm{R}$ & $20(11.3)$ & $31(8.9)$ & 1.28 & 0.35 & NS \\
\hline
\end{tabular}

\# $P u$, uncorrected $P$ values; $P c$, corrected $P$ values.

**Polymorphic amino acid positions analyzed: 6, 9, 11, 14, 16, 21, 24, 35, 45, 49, 52, 63, 66, 69, 73, 76, 77, 80, 90, 91, 94, 95, 97, 99, 103, 113, 114, 116, 138, 143, $147,152,156,163,170,173,175,177,178,180$.

Not shown are amino acid residues that were present in $90 \%$ or greater in both patient and control groups, or were present in $10 \%$ or fewer in both patient and control groups.

\section{Statistical analysis of HLA allele frequency}

The number of individuals carrying at least one copy of the allele were counted and compared with the number of individuals not carrying the allele for BA patients and controls, and similarly for comparisons between the mild and severe course. The significance for each allele was calculated using either a Chi square test or Fisher's exact test, as appropriate depending on the number of subjects in each contingency table. The resulting $P$ value for each allele ( $P u=$ uncorrected $P$ value) was corrected for multiple hypotheses testing using the false discovery rate (FDR) method of Benjamini and Yekutieli (2005), to control the expected number of Type I errors $(P c=$ corrected $P$ value). This method did not assume independence of tests as required by some FDR methods, and provided more power than the conservative Bonferroni correction.

\section{Epitope analysis}

Epitope analysis was performed using the $\mathrm{R}$ software package version 2.6.1 (available online at http://www.r-project.org). Combinations of 1-5 polymorphic amino acids at positions 8-93 of HLA molecules DRB1, DPB1 and DQB1, as well as combinations of up to 4 polymorphic residues at positions 2-182 of HLA molecules $\mathrm{A}, \mathrm{B}$ and $\mathrm{C}$ were considered possible epitopes. Polymorphic residues outside of these ranges are unlikely to influence peptide binding or $\mathrm{T}$ cell receptor interactions, and were not considered in this analysis. The number of individuals carrying at least one copy of the possible epitope was compared with the number of individuals not carrying it in both the BA group and controls, and similarly for comparisons between the mild and severe course. The epitope distribution among the patient and control populations was calculated by $2 \times 2$ contingency tables and analyzed with either Fisher's exact test or Pearson's chi-square test as appropriate. The $P$ value for each epitope was corrected for multiple comparisons using the false discovery rate method described by Benjamini and Yekutieli (2005) to control for Type I statistical errors.

\section{Results}

HLA alleles in BA

Allele analysis for HLA-A, -B, -C, -DRB1, -DPB1 and DQB1 in BA and racially-matched healthy controls did not identify any significant HLA association with BA (Additional file 1: Table S1). Analysis of each allele independently identified two possible alleles that may have been associated with BA: HLA-A*30:02 and -DRB1 
Table 5 HLA-DR single amino acid polymorphisms**

\begin{tabular}{|c|c|c|c|c|c|c|}
\hline Positions $^{\dagger}$ & Amino acid & Patients (178) n (\%) & Controls (684) n (\%) & Odds ratio & Pu value* & $P c$ value ${ }^{*}$ \\
\hline 9 & E & 103(57.87) & 207(30.26) & 1.9 & 0.56 & NS \\
\hline 9 & K & $150(84.27)$ & $291(42.54)$ & 2.0 & 0.81 & NS \\
\hline 10 & E & 118(66.29) & 242(35.38) & 1.9 & 0.29 & NS \\
\hline 10 & Q & 135(75.84) & $277(40.50)$ & 1.9 & 0.17 & NS \\
\hline 11 & D & $32(17.98)$ & $60(8.77)$ & 2.0 & 0.90 & NS \\
\hline 11 & G & $42(23.60)$ & 77(11.26) & 2.1 & 0.78 & NS \\
\hline 11 & L & $49(27.53)$ & $101(14.77)$ & 1.9 & 0.63 & NS \\
\hline 11 & $P$ & $54(30.34)$ & $97(14.18)$ & 2.1 & 0.64 & NS \\
\hline 11 & S & 118(66.29) & 242(35.38) & 1.9 & 0.29 & NS \\
\hline 12 & K & 118(66.29) & 242(35.38) & 1.9 & 0.29 & NS \\
\hline 12 & $\mathrm{~T}$ & 138(77.53) & $277(40.50)$ & 1.9 & 0.35 & NS \\
\hline 13 & $\mathrm{~F}$ & $25(14.04)$ & $53(7.75)$ & 1.8 & 0.66 & NS \\
\hline 13 & G & $42(23.60)$ & 77(11.26) & 2.1 & 0.78 & NS \\
\hline 13 & $\mathrm{H}$ & $42(23.60)$ & 76(11.11) & 2.1 & 0.72 & NS \\
\hline 13 & $\mathrm{R}$ & $49(27.53)$ & $101(14.77)$ & 1.9 & 0.63 & NS \\
\hline 13 & S & $50(28.09)$ & $96(14.04)$ & 2.0 & 1.00 & NS \\
\hline 13 & Y & 103(57.87) & 211(30.85) & 1.9 & 0.40 & NS \\
\hline 14 & E & $42(23.60)$ & 77(11.26) & 2.1 & 0.78 & NS \\
\hline 14 & K & 176(98.88) & $338(49.42)$ & 2.0 & 1.00 & NS \\
\hline 16 & $\mathrm{H}$ & $25(14.04)$ & $53(7.75)$ & 1.8 & 0.66 & NS \\
\hline 16 & Q & 176(98.88) & $339(49.56)$ & 2.0 & 1.00 & NS \\
\hline 25 & Q & $42(23.60)$ & 77(11.26) & 2.1 & 0.78 & NS \\
\hline 25 & R & 176(98.88) & $338(49.42)$ & 2.0 & 1.00 & NS \\
\hline 26 & $F$ & $39(21.91)$ & $76(11.11)$ & 2.0 & 0.94 & NS \\
\hline 26 & L & $45(25.28)$ & $73(10.67)$ & 2.4 & 0.31 & NS \\
\hline 26 & Y & 168(94.38) & $322(47.08)$ & 2.0 & 0.92 & NS \\
\hline 28 & D & $82(46.07)$ & $151(22.08)$ & 2.1 & 0.68 & NS \\
\hline 28 & E & 163(91.57) & $315(46.05)$ & 2.0 & 0.83 & NS \\
\hline 30 & $C$ & 18(10.11) & 23(3.36) & 3.0 & 0.17 & NS \\
\hline 30 & G & $32(17.98)$ & $60(8.77)$ & 2.0 & 0.90 & NS \\
\hline 30 & $\mathrm{H}$ & $42(23.60)$ & 77(11.26) & 2.1 & 0.78 & NS \\
\hline 30 & L & 159(89.33) & $316(46.20)$ & 1.9 & 0.24 & NS \\
\hline 31 & $F$ & $39(21.91)$ & $74(10.82)$ & 2.0 & 0.94 & NS \\
\hline 31 & 1 & 177(99.44) & $339(49.56)$ & 2.0 & 1.00 & NS \\
\hline 32 & $\mathrm{H}$ & $85(47.75)$ & $165(24.12)$ & 2.0 & 0.92 & NS \\
\hline 32 & Y & 158(88.76) & $318(46.49)$ & 1.9 & 0.10 & NS \\
\hline 33 & $\mathrm{H}$ & $50(28.09)$ & $96(14.04)$ & 2.0 & 1.00 & NS \\
\hline 33 & $N$ & 171(96.07) & $330(48.25)$ & 2.0 & 0.81 & NS \\
\hline 37 & L & $51(28.65)$ & $95(13.89)$ & 2.1 & 0.83 & NS \\
\hline 37 & $\mathrm{~N}$ & $75(42.13)$ & 150(21.93) & 1.9 & 0.71 & NS \\
\hline 37 & S & $78(43.82)$ & 147(21.49) & 2.0 & 0.85 & NS \\
\hline 37 & Y & $96(53.93)$ & $185(27.05)$ & 2.0 & 0.97 & NS \\
\hline 38 & L & $178(100.00)$ & $342(50.00)$ & 2.0 & 1.00 & NS \\
\hline 40 & $\mathrm{~F}$ & $178(100.00)$ & $342(50.00)$ & 2.0 & 1.00 & NS \\
\hline
\end{tabular}


Table 5 HLA-DR single amino acid polymorphisms** (Continued)

\begin{tabular}{|c|c|c|c|c|c|c|}
\hline 47 & $\mathrm{~F}$ & $123(69.10)$ & $243(35.53)$ & 1.9 & 0.64 & NS \\
\hline 47 & Y & 134(75.28) & $273(39.91)$ & 1.9 & 0.23 & NS \\
\hline 57 & D & $20(11.24)$ & $44(6.43)$ & 1.7 & 0.59 & NS \\
\hline 57 & $S$ & $58(32.58)$ & $100(14.62)$ & 2.2 & 0.43 & NS \\
\hline 57 & V & 169(94.94) & $322(47.08)$ & 2.0 & 0.84 & NS \\
\hline 58 & A & $32(17.98)$ & $63(9.21)$ & 2.0 & 0.90 & NS \\
\hline 58 & $\mathrm{E}$ & 175(98.31) & $340(49.71)$ & 2.0 & 0.34 & NS \\
\hline 60 & $S$ & $58(32.58)$ & $100(14.62)$ & 2.2 & 0.43 & NS \\
\hline 60 & Y & $175(98.31)$ & $333(48.68)$ & 2.0 & 0.76 & NS \\
\hline 67 & $\mathrm{~F}$ & $52(29.21)$ & 107(15.64) & 1.9 & 0.63 & NS \\
\hline 67 & I & $114(64.04)$ & $215(31.43)$ & 2.0 & 0.79 & NS \\
\hline 67 & L & $123(69.10)$ & $223(32.60)$ & 2.1 & 0.37 & NS \\
\hline 70 & D & $20(11.24)$ & $35(5.12)$ & 2.2 & 0.72 & NS \\
\hline 70 & Q & $125(70.22)$ & 228(33.33) & 2.1 & 0.41 & NS \\
\hline 70 & $\mathrm{R}$ & $130(73.03)$ & 254(37.13) & 2.0 & 0.76 & NS \\
\hline 71 & A & $43(24.16)$ & $89(13.01)$ & 1.9 & 0.64 & NS \\
\hline 71 & $\mathrm{E}$ & $47(26.40)$ & $78(11.40)$ & 2.3 & 0.36 & NS \\
\hline 71 & K & $58(32.58)$ & $120(17.54)$ & 1.9 & 0.57 & NS \\
\hline 71 & $\mathrm{R}$ & 139(78.09) & $264(38.60)$ & 2.0 & 0.82 & NS \\
\hline 73 & A & $77(43.26)$ & 134(19.59) & 2.2 & 0.37 & NS \\
\hline 73 & G & 170(95.51) & $321(46.93)$ & 2.0 & 0.55 & NS \\
\hline 74 & $E$ & $26(14.61)$ & 48(7.02) & 2.1 & 0.86 & NS \\
\hline 74 & L & $38(21.35)$ & $70(10.23)$ & 2.1 & 0.81 & NS \\
\hline 74 & Q & $42(23.60)$ & 77(11.26) & 2.1 & 0.78 & NS \\
\hline 74 & $\mathrm{R}$ & 153(85.96) & 299(43.71) & 2.0 & 0.64 & NS \\
\hline 77 & $N$ & $38(21.35)$ & 71(10.38) & 2.1 & 0.88 & NS \\
\hline 77 & $\mathrm{~T}$ & 175(98.31) & $338(49.42)$ & 2.0 & 0.70 & NS \\
\hline 78 & V & $49(27.53)$ & $90(13.16)$ & 2.1 & 0.77 & NS \\
\hline 78 & Y & 176(98.88) & $336(49.12)$ & 2.0 & 0.72 & NS \\
\hline 85 & V & $178(100.00)$ & $341(49.85)$ & 2.0 & 1.00 & NS \\
\hline 86 & G & $132(74.16)$ & $265(38.74)$ & 1.9 & 0.40 & NS \\
\hline 86 & V & $133(74.72)$ & $240(35.09)$ & 2.1 & 0.27 & NS \\
\hline
\end{tabular}

*Pu, uncorrected $P$ values; $P c$, corrected $\mathrm{P}$ values.

† Polymorphic amino acid positions $9,10,11,12,13,14,16,25,26,28,30,31,32,33,37,38,40,47,57,58,60,67,70,71,73,74,77,78,85,86$.

** Not displaying amino acid residues that were present in $90 \%$ or greater in both patient and control groups, or were present in $10 \%$ or fewer in both patient and control groups.

*15:01. However, neither was statistically significant when controlled for multiple comparisons. Secondly, we sought to determine if there was evidence for a HLA predominance in BA based on the severity of the disease: severe disease course (death or liver transplant in the first 2 years of life) vs. mild disease course (greater than 5 years of age and alive with their native liver). A comparison of severe and mild BA versus the controls yielded no significant association. Additionally, there were no significant differences between severe and mild BA when compared to each other (Additional file 2: Table S2).

\section{HLA shared epitope analysis in BA}

An epitope analysis was performed using all possible combinations of 1-4 amino acids for HLA-A (2,326,697 epitopes), HLA-B (3,129,345 epitopes), HLA-C (685,369 epitopes), and 1-5 amino acids for HLA-DRB1 (2,370, 369 epitopes), HLA-DQB1 (1,524,709 epitopes) and HLA-DPB1 (65,853 epitopes). Shown in Tables 2, 3, 4, 5, 6, 7 are the data for single amino acid combinations for each HLA group. None of 10,102,332 possible HLA epitopes was significantly different between BA patients and controls. Shared epitope analysis was also performed 
Table 6 HLA-DP single amino acid polymorphisms**

\begin{tabular}{|c|c|c|c|c|c|c|}
\hline Positions $^{\dagger}$ & Amino acid & Patients (111) n (\%) & Controls (91) n (\%) & Odds Ratio & Pu value* & $P c$ value* \\
\hline 8 & V & $59(53.15)$ & $39(42.86)$ & 1.2 & 0.15 & NS \\
\hline 9 & $\mathrm{H}$ & $8(7.21)$ & $11(12.09)$ & 0.6 & 0.33 & NS \\
\hline 9 & Y & $53(47.75)$ & $31(34.07)$ & 1.4 & 0.05 & NS \\
\hline 11 & L & $43(38.74)$ & $35(38.46)$ & 1.0 & 0.97 & NS \\
\hline 35 & Y & $27(24.32)$ & 10(10.99) & 2.2 & 0.01 & NS \\
\hline 36 & $A$ & $85(76.58)$ & $67(73.63)$ & 1.0 & 0.63 & NS \\
\hline 36 & V & 78(70.27) & $66(72.53)$ & 1.0 & 0.72 & NS \\
\hline 55 & A & $86(77.48)$ & $68(74.73)$ & 1.0 & 0.65 & NS \\
\hline 55 & $D$ & $75(67.57)$ & $64(70.33)$ & 1.0 & 0.67 & NS \\
\hline 56 & $A$ & 88(79.28) & $71(78.02)$ & 1.0 & 0.83 & NS \\
\hline 56 & E & $75(67.57)$ & $64(70.33)$ & 1.0 & 0.67 & NS \\
\hline 57 & D & $36(32.43)$ & $27(29.67)$ & 1.1 & 0.67 & NS \\
\hline 65 & L & $41(36.94)$ & $26(28.57)$ & 1.3 & 0.21 & NS \\
\hline 69 & E & 44(39.64) & $34(37.36)$ & 1.1 & 0.74 & NS \\
\hline 76 & V & $45(40.54)$ & $32(35.16)$ & 1.2 & 0.43 & NS \\
\hline 84 & D & $60(54.05)$ & $40(43.96)$ & 1.2 & 0.15 & NS \\
\hline 84 & G & $97(87.39)$ & $80(87.91)$ & 1.0 & 0.91 & NS \\
\hline 85 & $\mathrm{E}$ & $60(54.05)$ & $40(43.96)$ & 1.2 & 0.15 & NS \\
\hline 85 & G & 99(89.19) & 80(87.91) & 1.0 & 0.78 & NS \\
\hline 86 & A & $60(54.05)$ & $40(43.96)$ & 1.2 & 0.15 & NS \\
\hline 86 & P & 99(89.19) & $80(87.91)$ & 1.0 & 0.78 & NS \\
\hline 87 & M & 99(89.19) & 80(87.91) & 1.0 & 0.78 & NS \\
\hline 87 & V & $60(54.05)$ & $40(43.96)$ & 1.2 & 0.15 & NS \\
\hline
\end{tabular}

* $P u$, uncorrected $P$ values; $P c$, corrected $P$ values.

† Polymorphic amino acid positions $8,9,11,33,35,36,55,56,57,65,69,76,84,85,86,87 * *$ Not displaying amino acid residues that were present in $90 \%$ or greater in both patient and control groups, or were present in $10 \%$ or fewer in both patient and control groups.

comparing the severe and mild disease courses of BA. Single amino acid epitope analysis on polymorphic amino acid residues 2-182 for HLA-A, -B, -C and residues 8-93 for HLA-DRB1, -DPB1, and -DQB1 was performed in these 2 groups. No significant differences were identified between the severe and mild BA patients compared with controls. Finally, the severe and mild BA groups were compared to each other. 580 single amino acid epitopes across HLA-A, -B, -C, -DRB1, -DQB1, and -DPB1were compared between the severe and mild forms of BA and no significant differences between the two groups were identified (data not shown).

\section{Discussion}

This study encompasses the largest HLA allele frequency analysis for BA in the United States and is the first study to perform shared epitope analysis. When controlling for multiple comparisons, no HLA allele or shared epitope association was identified in BA. In previous HLA association studies, a serological phenotype technique was performed on small numbers of BA patients and identified potential HLA associations with BA HLA-B12
(Silveira et al. 1993) and HLA-B8 and -DR3(A-Kader et al. 2002). HLA genotyping is now based on DNAsequencing, which permits a greater number of HLA loci and alleles to be tested with increased accuracy. To that end, Donaldson et al. (Donaldson et al. 2002) genotyped 101 BA children and found no significant differences compared to controls. However, HLA-C was not analyzed and the genotyping was performed at low resolution, which limits the number of alleles that can be analyzed. Our study expands on this work as it measured all class I and class II HLA alleles by high resolution genotyping. A Japanese study of 392 BA patients and 828 controls analyzed 17 HLA-A, 19 HLA-B and 16 HLA-DR antigens (Yuasa et al. 2005). Significantly more BA patients expressed HLA-DR2 (DR15, DR16 in current nomenclature) (39\%) compared to controls $(30.4 \%)\left(p_{\mathrm{c}}=0.03\right.$; OR 1.46). Two locus analysis revealed that HLA-DR2 was not independently associated with BA but rather the combined expression of HLA-A24-B52DR2 was significantly greater in BA patients (14.9\%) versus controls (7.36\%) $(p=0.001$; OR 2.2), a phenomenon known as linkage disequilibrium. These results suggested 
Table 7 HLA-DQ single amino acid polymorphisms**

\begin{tabular}{|c|c|c|c|c|c|c|}
\hline Positions $^{\dagger}$ & Amino acid & Patients (178) n (\%) & Controls (342) n (\%) & Odds ratio & Pu value* & $P c$ value ${ }^{*}$ \\
\hline 9 & $\mathrm{~F}$ & $56(31.46)$ & $97(28.36)$ & 1.1 & 0.46 & NS \\
\hline 13 & A & $65(36.52)$ & 133(38.89) & 0.9 & 0.60 & NS \\
\hline 14 & $\mathrm{~L}$ & $57(32.02)$ & 123(35.96) & 0.9 & 0.37 & NS \\
\hline 26 & G & 69(38.76) & 149(43.57) & 0.9 & 0.29 & NS \\
\hline 26 & $L$ & 149(83.71) & 266(77.78) & 1.1 & 0.11 & NS \\
\hline 26 & Y & $65(36.52)$ & 133(38.89) & 0.9 & 0.60 & NS \\
\hline 28 & $S$ & $66(37.08)$ & $129(37.72)$ & 1.0 & 0.89 & NS \\
\hline 30 & $\mathrm{H}$ & $84(47.19)$ & $146(42.69)$ & 1.1 & 0.33 & NS \\
\hline 30 & $\mathrm{~S}$ & $66(37.08)$ & 129(37.72) & 1.0 & 0.89 & NS \\
\hline 30 & Y & 132(74.16) & 265(77.49) & 1.0 & 0.40 & NS \\
\hline 37 & I & $66(37.08)$ & 129(37.72) & 1.0 & 0.89 & NS \\
\hline 38 & A & $147(82.58)$ & 284(83.04) & 1.0 & 0.90 & NS \\
\hline 38 & V & $110(61.80)$ & $237(69.30)$ & 0.9 & 0.09 & NS \\
\hline 45 & $E$ & $60(33.71)$ & $120(35.09)$ & 1.0 & 0.75 & NS \\
\hline 46 & $E$ & $66(37.08)$ & $129(37.72)$ & 1.0 & 0.89 & NS \\
\hline 47 & $\mathrm{~F}$ & $66(37.08)$ & $129(37.72)$ & 1.0 & 0.89 & NS \\
\hline 52 & L & $66(37.08)$ & $129(37.72)$ & 1.0 & 0.89 & NS \\
\hline 53 & L & 148(83.15) & 277(80.99) & 1.0 & 0.55 & NS \\
\hline 53 & Q & $122(68.54)$ & $213(62.28)$ & 1.1 & 0.16 & NS \\
\hline 55 & L & $66(37.08)$ & $129(37.72)$ & 1.0 & 0.89 & NS \\
\hline 55 & $P$ & $94(52.81)$ & 192(56.14) & 0.9 & 0.47 & NS \\
\hline 55 & $\mathrm{R}$ & 130(73.03) & $231(67.54)$ & 1.1 & 0.20 & NS \\
\hline 57 & A & $95(53.37)$ & 179(52.34) & 1.0 & 0.82 & NS \\
\hline 57 & D & 132(74.16) & $261(76.32)$ & 1.0 & 0.59 & NS \\
\hline 57 & V & $54(30.34)$ & $100(29.24)$ & 1.0 & 0.79 & NS \\
\hline 66 & D & $83(46.63)$ & $167(48.83)$ & 1.0 & 0.63 & NS \\
\hline 67 & I & $83(46.63)$ & $167(48.83)$ & 1.0 & 0.63 & NS \\
\hline 70 & G & $110(61.80)$ & 194(56.73) & 1.1 & 0.27 & NS \\
\hline 70 & $\mathrm{R}$ & $150(84.27)$ & $280(81.87)$ & 1.0 & 0.49 & NS \\
\hline 71 & A & $57(32.02)$ & $122(35.67)$ & 0.9 & 0.41 & NS \\
\hline 71 & K & $66(37.08)$ & $129(37.72)$ & 1.0 & 0.89 & NS \\
\hline 71 & $\mathrm{~T}$ & 144(80.90) & 277(80.99) & 1.0 & 0.98 & NS \\
\hline 74 & A & $66(37.08)$ & $129(37.72)$ & 1.0 & 0.89 & NS \\
\hline 74 & $E$ & 144(80.90) & 277(80.99) & 1.0 & 0.98 & NS \\
\hline 74 & $S$ & 69(38.76) & 149(43.57) & 0.9 & 0.29 & NS \\
\hline 75 & $\mathrm{~L}$ & 144(80.90) & 277(80.99) & 1.0 & 0.98 & NS \\
\hline 75 & V & 116(65.17) & 244(71.35) & 0.9 & 0.15 & NS \\
\hline 77 & $\mathrm{R}$ & 105(58.99) & $225(65.79)$ & 0.9 & 0.13 & NS \\
\hline 77 & $\mathrm{~T}$ & 148(83.15) & $290(84.80)$ & 1.0 & 0.62 & NS \\
\hline 84 & $E$ & $122(68.54)$ & $213(62.28)$ & 1.1 & 0.16 & NS \\
\hline 84 & Q & 148(83.15) & $277(80.99)$ & 1.0 & 0.55 & NS \\
\hline 85 & $L$ & 148(83.15) & 277(80.99) & 1.0 & 0.55 & NS \\
\hline 85 & V & $122(68.54)$ & $213(62.28)$ & 1.1 & 0.16 & NS \\
\hline 86 & A & $114(64.04)$ & $206(60.23)$ & 1.1 & 0.40 & NS \\
\hline
\end{tabular}


Table 7 HLA-DQ single amino acid polymorphisms** (Continued)

\begin{tabular}{|c|c|c|c|c|c|c|}
\hline 86 & $E$ & 148(83.15) & 277(80.99) & 1.0 & 0.55 & NS \\
\hline 87 & $\mathrm{~F}$ & 69(38.76) & 113(33.04) & 1.2 & 0.19 & NS \\
\hline 87 & $L$ & 148(83.15) & 277(80.99) & 1.0 & 0.55 & NS \\
\hline 87 & Y & 69(38.76) & 134(39.18) & 1.0 & 0.93 & NS \\
\hline 89 & G & $122(68.54)$ & $213(62.28)$ & 1.1 & 0.16 & NS \\
\hline 89 & $\mathrm{~T}$ & 148(83.15) & 277(80.99) & 1.0 & 0.55 & NS \\
\hline 90 & I & $122(68.54)$ & $213(62.28)$ & 1.1 & 0.16 & NS \\
\hline 90 & $\mathrm{~T}$ & 148(83.15) & 277(80.99) & 1.0 & 0.55 & NS \\
\hline
\end{tabular}

* $P u$, uncorrected $P$ values; $P c$, corrected $P$ values.

† Polymorphic amino acid positions $9,13,14,23,26,27,28,30,37,38,45,46,47,52,53,55,56,57,60$ 66, 67, 70, 71, 74, 75, 77, 84, 85, 86, 87, 89, 90.

** Not displaying amino acid residues that were present in $90 \%$ or greater in both patient and control groups, or were present in $10 \%$ or fewer in both patient and control groups.

that the gene for BA susceptibility is at a locus in close proximity to the HLA locus.

One might argue that a larger BA population needed to be studied in order to identify a HLA allele association. Indeed, our power calculations to identify a $5 \%$ increase in a specific HLA allele frequency in BA versus controls (i.e. $15 \%$ BA versus $10 \%$ controls) would have required a sample size of 316 BA patients. However, the shared epitope analysis is extremely sensitive at identifying potential HLA associations and the evaluation of $>10$ million epitopes most certainly would have identified an association (Freed et al. 2011; Karp et al. 2010). For example, Freed et al. performed shared epitope analysis on patients with rheumatoid arthritis and assessed all possible combinations of up to 5 amino acids within the peptide binding groove of HLA-DRB1 (Freed et al. 2011). Several HLA-DR4 alleles have previously been linked with rheumatoid arthritis, with strong associations with DRB1*04:01, *04:04, and *04:05, and weaker associations with DRB1*01:01, *01:02, *10:01, and *14:02. These disparate alleles had been hypothesized to contribute to rheumatoid arthritis via the presence of a shared epitope at the peptide-binding groove. Of the $>2$ million epitopes examined, LA ${ }^{67,74}$ (leucine at position 67 and alanine at position 74) exhibited the highest correlation with rheumatoid arthritis susceptibility $\left(P=2 \times 10^{-20}\right)$. This same group is presently analyzing for shared epitopes in diabetes and chronic hepatitis $\mathrm{C}$ infection in order to predict disease associations and prognoses.

One limitation of our study is that we did not analyze the HLA-DRB3, DRB4, DRB5, DQA1, or DPA1 alleles. We chose not to analyze these few alleles because the literature examining disease associations with these loci is sparse and these loci are linked to other loci that were tested for and therefore would have identified the association. For example, DQA1 is tightly linked to DQB1, so if there were a DQA1 link we would have seen it in the DQB1 analysis. This is similarly true for DRB3, DRB4 and
DRB5, albeit they are less tightly linked (Thorsby \& Lie 2005; Gough \& Simmonds 2007; Shiina et al. 2004).

Based on our findings showing no HLA or shared epitope association in BA, one must consider other possible genetic influences that alter the immune response. Genome-wide association studies have recently been performed to identify disease-specific genetic associations. A Chinese study (Garcia-Barcelo et al. 2010) genotyped nearly half a million single nucleotide polymorphisms (SNPs) in 200 BA patients and 481 controls and identified a strong association of BA with the SNP rs17095355 on chromosome 10q24. Two genes in the region of this SNP include X-prolyl aminopeptidase P1(XPNPEP1) and adducin 3 (ADD3). XPNPEP1 is expressed in biliary epithelia and is involved in the metabolism of inflammatory mediators. Genetic defects of XPNPEP1 could result in deregulation of control of the inflammatory response present in BA. ADD3 is expressed in hepatocytes and biliary epithelia and is involved in the assembly of spectrin-actin membrane protein networks at sites of cell to cell contact. Defects in this gene could theoretically increase fibrosis. Importantly, if there was an HLA association with BA in this Chinese population, it would have been identified within the GWAS analysis detailed above.

Future studies of genetic links to BA that involve alterations of the immune response should include investigations into defects in regulatory $\mathrm{T}$ cells (Treg) that would allow inflammation to proceed unchecked. To that end, we have recently identified significant deficits in peripheral blood Treg frequencies in BA infants at the time of diagnosis compared to age-matched controls (Brindley et al. 2012). In the murine model of BA, Lages et al. (2012) showed that adoptive transfer of total CD4 ${ }^{+} \mathrm{T}$ cells, but not Treg-depleted $\mathrm{CD}_{4}{ }^{+} \mathrm{T}$ cells, into RRV-infected BA mice was associated with increased survival and decreased bile duct targeted inflammation, suggesting that Tregs protect from bile duct damage. Another avenue of future research into the pathogenesis of BA should in- 
clude investigations into the possibility that BA is an autoinflammatory disease. Autoinflammatory diseases are not usually HLA-linked and are characterized by exaggerated innate immune responses (Goldbach-Mansky 2012; Rigante 2012).

\section{Additional files}

Additional file 1: Frequencies of HLA alleles.

Additional file 2: Table S2. HLA allele frequencies of mild versus severe BA.

\section{Abbreviations}

ADD3: Adducin 3; BA: Biliary Atresia; CHiLDREN: Childhood Liver Disease Research and Education Network; SBT: Sequence based typing; GWAS: Genome-wide association studies; HLA: Human leukocyte antigen; SNPs: Single Nucleotide Polymorphisms; XPNPEP1: X-prolyl aminopeptidase P1.

\section{Competing interests}

No conflicts of interest with any of the authors.

\section{Authors' contributions}

CM, RS and PR designed the study, analyzed the data, contributed to the manuscript writing and are ChiLDREN members; KA, MA and BF performed all of the molecular genetic studies, analyzed the data and assisted in manuscript writing. All authors read and approved the final manuscript.

\section{Disclaimer}

This manuscript was not prepared in collaboration with other ChiLDREN investigators and does not necessarily reflect the opinions or views of ChiLDREN or the NIDDK.

\section{Acknowledgement}

We greatly appreciate the ChiLDREN investigators, coordinators, and families who participated, as they made this work possible.

\section{Financial support}

1. R56 DK087691 National Institutes of Health, NIDDK (PI: Mack CL)

2. 2U01DK062453 National Institutes of Health, NIDDK; Colorado Center for Childhood Liver Disease Research and Education Network (PI: Sokol RJ) and U01 DK062456 (DCC University of Michigan)

3. UL1 TR000154-05 National Institutes of Health, NCATS; Colorado Clinical and Translational Sciences Institute (PI: Sokol, RJ)

\section{Author details}

'Departments of Medicine and Immunology, Division of Allergy and Clinical Immunology, University of Colorado School of Medicine, Aurora, CO 80045, USA. ${ }^{2}$ Clinimmune Labs, 12635 E Montview Blvd. Suite 300, Aurora, CO 80045, USA. ${ }^{3}$ Department of Pediatrics, Division of Pediatric Gastroenterology, Digestive Health Institute, Children's Hospital Colorado, Hepatology and Nutrition, 13123 East 16th Ave. B290, Aurora, CO 80045, USA. ${ }^{4}$ The Liver Center, University of CA San Francisco, 500 Parnassus Ave, San Francisco, CA 94143, USA

Received: 23 November 2012 Accepted: 4 February 2013

Published: 8 February 2013

\section{References}

Abbas AK, Lichtman AH (2005) The Major Histocompatibility Complex. Cellular and Molecular Immunology 32(5):65-80

A-Kader HH, El-Ayyouti M, Hawas S, Abdalla A, Al-Tonbary Y, Bassiouny M, Boneberg A, El-Sallab S (2002) HLA in Egyptian children with biliary atresia. Pediatr 141:432

Benjamini Y, Yekutieli D (2005) Quantitative trait loci analysis using the false discovery rate. Genetics 171:783-790

Brindley SM, Martin A, Tucker RM, Karrer F, Fontenot AP, Mack CL (2012) Cytomegalovirus-specific $T$ cell reactivity in biliary atresia at the time of diagnosis is associated with deficits in regulatory T cells. Hepatology 55 (4):1130-1138

Donaldson PT, Clare M, Constantini PK, Hadzic N, Mieli-Vergani G, Howard E, Kelley D (2002) HLA and cytokine gene polymorphisms in biliary atresia. Liver 22:213-219

Freed BM, Schuyler RP, Aubrey MT (2011) Association of the HLA-DRB1 epitope LA67, 74 with rheumatoid arthritis and citrullinated vimentin binding. Arthritis Rheum 63(12):3733-3739

Garcia-Barcelo MM et al (2010) Genome-wide association study identifies a susceptibility locus for biliary atresia on 10q24.2. Hum Mol Genet 19 (14):2917-2925

Goldbach-Mansky R (2012) Immunology in clinic review series; focus on autoinflammatory diseases: update on monogenic autoinflammatory diseases: the role of interleukin (IL)-1 and an emerging role for cytokines beyond IL-1. Clin Exp Immunol 167(3):391-404

Gough SCL, Simmonds MJ (2007) The HLA region and autoimmune disease: associations and mechanisms of action. Curr Genomics 8:453-465

Gregersen PK, Silver J, Winchester RJ (1987) The shared epitope hypothesis. An approach to understanding the molecular genetics of susceptibility to rheumatoid arthritis. Arth Rheum 30:1205

Karp DR, Marthandan N, Marsh SG, Ahn C, Arnett FC, DeLuca DS et al (2010) Novel sequence feature variant type analysis of the HLA genetic association in systemic sclerosis. Hum Mol Genet 19:707-719

Klareskog L, Alfredsson L, Rantapaa-Dahlqvist S, Berglin E, Stolt P, Padyukov L (2004) What precedes the development of rheumatoid arthritis. Ann Rheum Dis 63(suppl 2):ii28

Lages CS, Simmons J, Chougnet CA, Miethke AG (2012) Regulatory T cells control the CD8 adaptive immune response at the time of ductal obstruction in experimental biliary atresia. Hepatology 56(1):219-227

Mack CL (2007) The pathogenesis of biliary atresia: evidence for virus-induced autoimmune disease Semin Liver Dis 27(3):233-242

Mack CL, Tucker RM, Lu BR, Sokol RJ, Fontenot AP, Ueno Y, Gill RG (2006) Cellular and humoral autoimmunity directed at bile duct epithelia in murine biliary atresia. Hepatology 44(5):1231-1239

Mack CL, Falta MT, Sullivan AK, Karrer F, Sokol RJ, Freed BM, Fontenot AP (2007) Oligoclonal Expansions of CD4 ${ }^{+}$and $\mathrm{CD}^{+} \mathrm{T}$ Cells in the Target Organ of Patients with Biliary Atresia. Gastroenterology 133(1):278-287

Rigante D (2012) The fresco of autoinflammatory diseases from the pediatric perspective. Autoimmun Rev 11(5):348-356

Shiina T, Inoko H, Kulski JK (2004) An update of the HLA genomic region, locus information and disease associations:2004. Tissue Antigens 64:631-649

Shivakumar P, Sabla G, Mohanty S, McNeal M, Ward R, Stringer K, Caldwell C, Chougnet C, Bezerra JA (2007) Effector role of neonatal hepatic CD8+ lymphocytes in epithelial injury and autoimmunity in experimental biliary atresia. Gastroenterology 133(1):268-277

Silveira TR, Salzano FM, Donaldson PT, Mieli-Vergani G, Howard ER, Mowat AP (1993) Association between HLA and extrahepatic biliary atresia. J Pediatr Gastroenterol Nutr 16:114-117

Sokol RJ, Mack CL, Narkewicz MR, Karrer FM (2003) Pathogenesis and outcome of biliary atresia: current concepts. J Pediatr Gastroenterol Nutr 37:4-21

Stern JL, Brown JH, Jardetzky TS, Gorga JC, Urban RG, Strominger JL et al (1994) Crystal structure of the human class II MHC protein HLA-DR1 complexed with an influenza virus peptide. Nature 368(6468):215-221

Thorsby E, Lie BA (2005) HLA associated genetic predisposition to autoimmune diseases: genes involved and possible mechanisms. Transpl Immunol 14:175182

Yuasa T, Tsuji H, Kimura S, Niwa N, Yurugi K, Egawa H, Tanaka K, Maruya E, Saji HO, Asano H, Maekawa T (2005) Human leukocyte antigens in Japanese patients with biliary atresia: retrospective analysis of patients who underwent living donor liver transplantation. Hum Immunol 66:295-300

doi:10.1186/2193-1801-2-42

Cite this article as: Mack et al:: Lack of HLA predominance and HLA

shared epitopes in biliary Atresia. SpringerPlus 2013 2:42. 